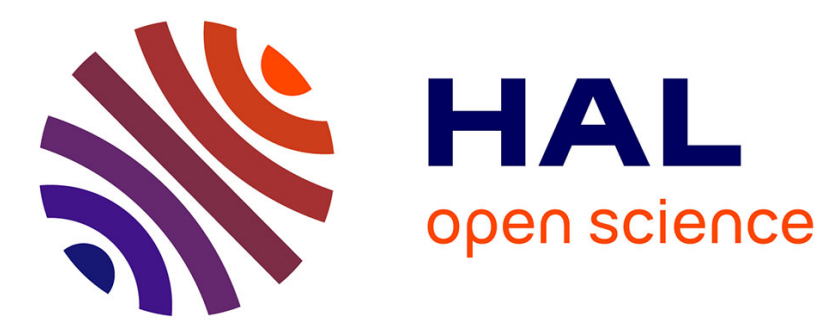

\title{
L'apport ignoré de la sociologie historique et réflexive des sciences
}

Marc Joly, Julien Larregue

\section{To cite this version:}

Marc Joly, Julien Larregue. L'apport ignoré de la sociologie historique et réflexive des sciences. Zilsel : science, technique, société, 2020, N7 (2), pp.442. 10.3917/zil.007.0442 . halshs-03092190

\section{HAL Id: halshs-03092190 \\ https://shs.hal.science/halshs-03092190}

Submitted on 28 Aug 2021

HAL is a multi-disciplinary open access archive for the deposit and dissemination of scientific research documents, whether they are published or not. The documents may come from teaching and research institutions in France or abroad, or from public or private research centers.
L'archive ouverte pluridisciplinaire HAL, est destinée au dépôt et à la diffusion de documents scientifiques de niveau recherche, publiés ou non, émanant des établissements d'enseignement et de recherche français ou étrangers, des laboratoires publics ou privés. 


\section{L'apport ignoré de la sociologie historique et réflexive des sciences}

Marc Joly ${ }^{1}$ et Julien Larregue ${ }^{2}$

À propos de Laurent Cordonier, La nature du social. L'apport ignoré des sciences cognitives, Paris, Presses universitaires de France, 2018, 374 pages.

L'ouvrage de Laurent Cordonier, publié aux Presses universitaires de France sous la direction scientifique de Gérald Bronner (côtoyé par l'auteur à l'Université Paris-Diderot) et issu d'une thèse de doctorat soutenu à l'Université de Lausanne sous la direction de Laurence Kaufmann, a pour ambition affichée d'ouvrir la sociologie à d'autres sciences, concernées par l'étude de la « cognition » ou, plus largement, de la «nature humaine » (psychologie, neurosciences, primatologie, etc.).

La démarche de l'auteur, actuellement chercheur post-doctorant, se déploie en deux temps. Dans un premier temps (les quatre premiers chapitres), il présente sa conception des rapports entre les « sciences naturelles » et les « sciences sociales », puis dresse une sorte d'état des lieux, de la primatologie à l'anthropologie cognitive en passant par la psychologie cognitive et la linguistique chomskienne, d'où il résulte, en particulier, que la « cognition sociale » des êtres humains engloberait davantage que leur seule capacité à se représenter les états mentaux constitutifs des actions d'autrui (leur bien mal nommée «théorie de l'esprit»). Dans un second temps (chapitres 5 et 6), il s'efforce de mettre en avant ce que ces différentes sciences et approches pourraient apporter aux recherches menées en sociologie.

Il convient de saluer le sérieux du travail livré par l'auteur. Il fait beaucoup d'efforts pour être compris et son écriture est toujours limpide. Si, pour une grande part, il résume des travaux et des résultats relativement bien connus, mobilisant une impressionnante littérature (quasi exclusivement de langue anglaise), il n'hésite pas à formuler des propositions originales. Il n'en reste pas moins, et c'est paradoxal, que son étude est extrêmement difficile à évaluer. Ce qui est en cause, de notre point de vue de chercheurs attachés à pratiquer une sociologie historique et réflexive des sciences ${ }^{3}$, c'est la posture même de l'auteur : à défaut de soumettre à un examen proprement sociologique les disciplines ou les courants qu'il nous présente, censés féconder la sociologie, il s'est condamné à reprendre à son compte certains de leurs biais et impensés. Quel est l'objet exact de son étude ? Quels principes ont gouverné ses choix ? Quelle est sa vision de la sociologie ? Sur toutes ces questions, règne un flou qui a gêné notre lecture. Nous allons essayer d'expliciter pourquoi et de clarifier, chemin faisant, les termes de certains problèmes théoriques.

Nous débuterons par une analyse des choix terminologiques opérés par Laurent Cordonier, en montrant qu'ils procèdent assez largement d'une récupération non réflexive des catégories de pensée des principaux représentants des domaines de recherche dont il tire son inspiration. Nous analyserons dans une deuxième partie le modèle de la «cognition sociale » défendu par l'auteur, en soulignant ses limites : caractère formaliste, vision non processuelle des actions et pensées humaines, conceptualisation lacunaire des interdépendances entre facteurs sociologiques, psychologiques et biologiques. Nous examinerons enfin la pertinence et les apports concrets de cette approche théorique du point de vue de la compréhension des faits sociologiques à partir, en particulier, des développements consacrés par l'auteur au concept d'habitus.

\section{« Sciences cognitives » et « sciences de la nature humaine » : de quoi s'agit-il ?}

Commençons par un problème d'ordre terminologique. Laurent Cordonier parle tour à tour de «sciences cognitives » et de «sciences de la nature humaine ». Or ces dénominations renvoient à des réalités qui ne s'équivalent pas. L'auteur, qui ne l'ignore sans doute pas, se garde de toute explicitation.

L'appellation « sciences cognitives » est notoirement ambigüe. L'objet de ces sciences, selon une définition répandue, est de « décrire, d'expliquer et le cas échéant de simuler les principales dispositions et capacités de l'esprit humain - langage, raisonnement, perception, coordination motrice, planifica-

\footnotetext{
${ }^{1}$ CNRS, Printemps, UVSQ-CNRS, marc.joly@uvsq.fr.

${ }^{2}$ ISP, ENS Paris-Saclay-CNRS, julien.larregue@ens-cachan.fr.

${ }^{3}$ Pour une utile présentation de cette tradition de recherche, voir Johan Heilbron, «Pour une sociologie historique et réflexive des sciences humaines et sociales », Revue d'histoire des sciences humaines, $\mathrm{n}^{\circ} 30,2017$, p. 277-288.
} 
tion ${ }^{4}$. Il est cependant possible d'affirmer que l'utilisation de l'étiquette en cause, au même titre que celle d' "économie », "est plus l'indice d'un travail intense - mais jamais achevé - de construction sociale du groupe que la preuve d'une unité réelle bien établie, fondée sur un corpus de connaissances clairement délimité et constitué, des normes scientifiques, des procédures professionnelles partagées. ${ }^{5}$

Cela ne signifie pas que les sciences cognitives soient insaisissables et, partant, hors de portée de l'analyse sociologique : elles dessinent un champ identifiable, qui s'est transformé (la vogue de la " cognition incarnée ${ }^{6}$ » ayant par exemple quelque peu relégué au second plan calcul, traitement de l'information et représentation), et dont la formation originelle a partie liée tout à la fois avec l'essor prodigieux de la simulation informatique et avec les dynamiques de transformation propres à certaines disciplines universitaires nord-américaines, en particulier la psychologie et la linguistique, «révolutionnées » de concert, à partir de la fin des années 1950, par des jeunes agents hostiles au behaviorisme (Jerome Bruner, Noam Chomsky, etc.).

Le champ des « sciences cognitives » s'est traduit par les oppositions, désaccords et incompréhensions qui sont souvent le lot des échanges entre disciplines : les neurobiologistes, lorsqu'ils s'y investiront, le feront surtout en réaction à l'approche informatique des fondateurs, pour mieux ramener le fonctionnement neurophysiologique du cerveau sur le devant de la scène ${ }^{7}$. Il a par ailleurs pour caractéristique d'avoir été fortement irrigué par les problématiques de la "philosophie de l'esprit » et, plus largement, de toute la tradition philosophique classique : des philosophes de l'« esprit » et du « langage » n'ont pas tardé, en effet, à tenter de lui conférer un semblant d'unité via la réactivation des problèmes de la « représentation ${ }^{8}$ ou des rapports entre l'âme et le corps. Ce champ, auquel se superposera largement, au fil du temps, le champ lui-même transdisciplinaire des neurosciences, réunit ainsi des ingénieurs en informatique, des psychologues, des linguistes, des neurobiologistes et des philosophes, auxquels se sont joints, plus ou moins ponctuellement, des politistes, des économistes et des anthropologues.

Par comparaison, les «sciences de la nature humaine » ne se rapportent pas à un «programme », à un « paradigme», et encore moins à une supposée «révolution» (le «cognitivisme») ; elles ne se rattachent pas à des initiatives institutionnelles qu'il serait possible de circonscrire avec précision (fondations, revues, instituts, centres de recherche voire facultés, etc.). À se cantonner à la dimension biologique, on pourrait y faire entrer, parmi d'autres disciplines, l'éthologie, la biologie évolutive, la microbiologie, l'épigénétique, l'éthologie, l'anthropologie physique ou encore la paléontologie. Mais tous ces champs de recherche n'étant pas discutés dans l'ouvrage de Laurent Cordonier, on est bien en peine d'identifier une ligne directrice claire.

Il y a toutefois un point commun entre ces deux appellations et les configurations - relativement délimitée sur les plans paradigmatique et institutionnel, pour la première, large et floue pour la seconde auxquelles elles renvoient: dans les deux cas, sauf exception, les sociologues sont rejetés vers l'extérieur. Certes, dira-t-on, l'expression «sciences de la nature humaine » permet simplement de désigner l'ensemble des sciences humaines non sociales. Commode, elle n'en charrie pas moins des dichotomies (les oppositions «nature $/$ / culture» ou «sciences de la nature $/$ / «sciences de l'esprit ») qui ne manquent pas de paraitre arbitraires à la lumière de la sociologie des sciences. Ce n'est pas un hasard si les «sciences cognitives » excluent de leur programme la sociologie comme science des processus sociaux, comme pôle majeur de «l'étude scientifique de l'homme» et comme

\footnotetext{
${ }^{4}$ Daniel Andler, «Introduction. Calcul et représentations : les sources », in Daniel Andler (dir.), Introduction aux sciences cognitives, Paris, Gallimard, 1992, p. 9.

${ }^{5}$ Frédéric Lebaron, La croyance économique: les économistes entre science et politique, Paris, Seuil, 2000, p. 17-18.

${ }^{6}$ Voir Arthur M. Glenberg, «Embodiment as a unifying perspective for psychology », WIREs Cognitive Science, vol. 1, 2010, p. 586-596 ; Andrew D. Wilson, Sabrina Golonka, « Embodied cognition is not what you think it is », Frontiers in Psychology, vol. 4, 2013, p. 1-13.

${ }^{7}$ Brigitte Chamak, «The emergence of cognitive science in France: A comparison with the USA », Social Studies of Science, vol. 29, $\mathrm{n}^{\circ}$ 5, 1999, p. 643-684.

${ }^{8}$ «Il est indéniable que le centre de gravité des sciences cognitives se situe sur l'axe, traditionnel dans la philosophie occidentale, de la représentation, vue comme rapport entre une réalité indépendante et un sujet à la recherche d'une image fidèle de cette réalité, et plus ou moins bien équipé pour l'obtenir. » (Daniel Andler, «Introduction. Calcul et représentations : les sources », op. cit., p. 43.)

${ }^{9}$ Émile Durkheim, L'évolution pédagogique en France, Paris, Presses universitaires de France, 1999 [1938], p. 283.
} 
théorie de la connaissance (et donc de la cognition) en vertu de présupposés épistémologiques et d'enjeux institutionnels dont la mise au jour montrerait, selon nous, qu'ils procèdent d'une série de coups de force tout à fait discutables ${ }^{10}$. Pour le dire autrement, si la sociologie pâtit peut-être de "l'apport ignoré des sciences cognitives", il y a d'abord eu, assurément, ignorance délibérée par lesdites "sciences cognitives 》 des apports de l'étude socio-historique des catégories de pensée, des logiques de classement et des normes de sensibilité - c'est-à-dire de la cognition et de l'affectivité qui structure le paradigme sociologique classique, d'Émile Durkheim à Pierre Bourdieu en passant par Norbert Elias. Cette mise à l'écart ne concerne au demeurant pas la seule sociologie. Elle affecte également la psychanalyse et, à un degré moindre, dans le domaine renouvelé de la psychologie cognitive, le structuralisme génétique d'un pionnier comme Jean Piaget. Un spécialiste de ce dernier s'étonnait en ces termes : «Il peut [...] paraître tout à fait paradoxal que l'on s'interroge ici sur les rapports entre l'ouvre de Jean Piaget et les sciences cognitives : cette æuuvre est en grande partie une science cognitive. ${ }^{11} \mathrm{Ce}$ paradoxe vaut plus encore pour la sociologie.

Mais encore aurait-il fallu, pour que les lecteurs puissent apprécier les processus de succession et les logiques des prises de position en jeu, ne serait-ce que sous la forme d'un bref aperçu, que l'auteur ait mis en œuvre, préalablement à toute réflexion sur l'ouverture de la sociologie à l'égard d'autres disciplines et approches scientifiques, une démarche de sociologie des sciences. Nous serions assez tentés de dire qu'il y a là une étape absolument incontournable. Au fond, un sociologue, lorsqu'il s'interroge sur les rapports entre sa discipline et d'autres disciplines, n'est pas libre : il doit d'abord faire la sociologie de sa propre science et des autres sciences, et, le cas échéant, donner une idée de l'espace de relations objectives dans lequel elles se situent. Plus exactement, il n'est sans doute pas de meilleure façon pour un sociologue de se positionner vis-à-vis des autres sciences que d'en faire la sociologie après avoir mené sa propre socioanalyse. Il ne s'agit pas seulement, partant, d'envisager les choses sous l'angle de l'histoire sociale des institutions d'enseignement et de recherche, des disciplines, des pratiques savantes et des concepts. Il importe de prendre la mesure, qui plus est, des contraintes logiques inhérentes au régime de pensée multidimensionnel de l'humanité que la sociologie comme discipline a été amenée à parachever à la fin du $19^{\mathrm{e}}$ siècle $^{12}$.

Une telle approche est à l'opposé de la vision de Laurent Cordonier, qui prête aux sciences biologiques et psychologiques de la cognition humaine la fonction d' «évaluer et [d']enrichir l'esprit sociologique » (p. 255) sans envisager franchement que les sciences sociales puissent alimenter en retour les protocoles méthodologiques et l'épistémologie des «sciences naturelles ». Cette dernière expression, de même que le vocable naturalisme, aurait d'ailleurs mérité d'être historicisée ${ }^{13}$. Il reste qu'on gagne probablement en clarté lorsqu'on se borne à situer les sciences sociologiques par rapport aux sciences biologiques et psychologiques, et qu'on tâche de reconstituer et de mettre en perspective le système de leurs interrelations : produit de l'histoire, il obéit à des contraintes logiques indissociables des caractéristiques immanentes de l'humanité.

Il n'est évidemment pas possible, dans l'espace de cette note critique, de pallier l'absence de mise en œuvre d'une analyse réflexive et historiciste des sciences. Nous remarquerons seulement que le flou des appellations entretenu par l'auteur empêche par exemple de saisir la complexité des recomposi-

\footnotetext{
${ }^{10}$ Le projet consistant à mettre en relation sur nouveaux frais les processus mentaux avec le monde physique via les notions d'information, de computation et de rétroaction n'imposait pas nécessairement l'exclusion ou la subalternisation des sciences sociales. Seule pourrait nous éclairer une enquête sociologique attentive aux progrès technologiques et aux luttes et rivalités (entre agents dotés de propriétés et de ressources différentes) ayant favorisé cette mise en relation selon certaines modalités pratiques et discursives.

${ }^{11}$ Jean-Jacques Ducret, «Piaget et les sciences cognitives », Intellectica. Revue de l'Association pour la Recherche Cognitive, $\mathrm{n}^{\circ}$ 33, 2001, p. 209.

${ }^{12}$ Voir Marc Joly, Après la philosophie. Histoire et épistémologie de la sociologie européenne, Paris, CNRS Éditions, 2020, p. 25-27, p. 53-56, p. 86-90.

${ }^{13}$ Durkheim parlait ainsi de «naturalisme sociologique» en un sens tout à fait précis, faisant justice à l'«originalité » et à la «complexité» irréductible du «règne social», dans une perspective comtienne d'épistémologie différenciée, tout en consacrant l'entrée de celui-ci dans le royaume du déterminisme universel, parmi l'ensemble des «êtres de la nature»: «Cette méthode est naturaliste puisqu'elle prescrit au sociologue l'attitude mentale qui est de règle dans les sciences naturelles. Mais elle n'est pas naturaliste au sens ordinaire du mot, puisqu'elle ne tend pas à résorber le règne social dans les autres règnes de la nature, mais, au contraire, exige qu'on lui laisse toute son originalité. Le naturalisme qu'elle pratique est essentiellement sociologique » (Émile Durkheim, «La sociologie en France au 19 ${ }^{\mathrm{e}}$ siècle » [1900], dans La science sociale et l'action, introduction de Jean-Claude Filloux, Paris, Presses Universitaires de France, 2010, p. 136).
} 
tions du champ scientifique de ces dernières décennies. Aux États-Unis et en Grande-Bretagne, de plus en plus de sociologues, d'économistes, de criminologues et de politistes mobilisent des données génétiques et neuroscientifiques afin d'aboutir à une explication biosociale des comportements humains ${ }^{14}$. Ce mouvement, qui s'est notamment matérialisé dans des collaborations interdisciplinaires entre des chercheurs en sciences sociales et en neurosciences ${ }^{15}$, a également donné lieu à un processus d'institutionnalisation, comme en témoigne le Social Science Genetic Association Consortium, créé en 2011 pour permettre la mise en commun des données nécessaires à la réalisation des coûteuses « genome-wide association studies » (GWAS). Les ressorts, les implications et les limites de ces diverses entreprises sont ignorés par Laurent Cordonier. On peut se demander, de même, pourquoi il ne consacre aucun développement à la recherche menée en épigénétique, qui constitue, pour d'aucuns, l'une des principales voies susceptibles de favoriser une réconciliation entre sciences sociales et sciences biologiques ${ }^{16}$.

\section{Les points aveugles du « naturalisme social »}

Laissant de côté ces mouvements scientifiques contemporains - et bien d'autres encore -, Laurent Cordonier s'attache à faire valoir la supériorité du programme théorique «naturaliste » qui a sa faveur par rapport aux deux autres «naturalismes » sur lesquels portent ses critiques, quand bien même plus grand monde, aujourd'hui, ne songe à s'en réclamer.

Dans le premier chapitre, il renvoie en effet dos à dos le «naturalisme réductionniste » (p. 23) de la sociobiologie d'Edward $\mathrm{O}$. Wilson et le «naturalisme analogique » (p. 32) de la mémétique de Richard Dawkins. La sociobiologie wilsonienne est tellement discréditée que l'auteur n'a guère qu'une essayiste controversée - Peggy Sastre - à se mettre sous la dent à titre de manifestation contemporaine $^{17}$. Quant à la mémétique, il démontre sans mal son ineptie. Se dissociant ainsi de deux options très pauvres pour comprendre les phénomènes sociaux, il indique que "l'objet de [son] ouvrage », par contraste, est un «naturalisme social » qui vise à «intégrer certaines connaissances » des « sciences naturelles » «aux élaborations théoriques et aux démarches explicatives des sociologues » (p. 39). Or le «naturalisme social développé par les sociologues et philosophes Laurence Kaufmann et Fabrice Clément » (p. 16), dit "intégratif », est précisément l'approche dans laquelle il affirme se reconnaître en introduction. De manière assez étonnante, il n'en est plus explicitement question dans ce premier chapitre. Le lecteur qui n'est pas familier des travaux de Kaufmann et Clément doit attendre une cen-

\footnotetext{
${ }^{14}$ Voir Catherine Bliss, Social by Nature: The Promise and Peril of Sociogenomics, Stanford, Stanford University Press, 2018 ; Julien Larregue, " "C'est génétique" : ce que les twin studies font dire aux sciences sociales », Sociologie, vol. $9, \mathrm{n}^{\circ} 3,2018$, p. 285-304.

${ }^{15}$ Voir Felicity Callard, Des Fitzgerald, Rethinking Interdisciplinarity across the Social Sciences and Neurosciences, Basingstoke, Palgrave Macmillan, 2016.

${ }^{16}$ Voir Michel Dubois, Catherine Guaspare, Séverine Louvel, « De la génétique à l'épigénétique : une révolution "post-génomique" à l'usage des sociologues », Revue française de sociologie, vol. 59, n 1, 2018, p. 71-98; Maurizio Meloni, Giuseppe Testa, «Scrutinizing the epigenetics revolution », BioSocieties, vol. 9, n 4, 2014, p. 431-456.

${ }^{17}$ La réfutation multidisciplinaire de Patrick Tort et al. (Misère de la sociobiologie, Paris, Presses universitaires de France, 1985) reste largement valable. Il existe, cela dit, des héritiers de la sociobiologie qui ont su se libérer de ses impasses conceptuelles les plus criantes. Le primatologue et anthropologue canadien Bernard Chapais a ainsi mis en œuvre une démarche de recherche exceptionnellement patiente et réfléchie : ayant opéré un long détour par la primatologie dans le but de mieux comprendre les bases biologiques des comportements humains, il s'est ensuite formé à l'anthropologie culturelle pour livrer l'une des grandes synthèses de ces dernières décennies : Bernard Chapais, Aux origines de la société humaine. Parenté et évolution, Paris, Seuil, 2017. Ce dont il est question - la mise au jour, sous le nom de «nature humaine », de constantes au principe de la variabilité des comportements et des institutions - renvoie à des processus qui se déroulent sur des millions d'années, combinant évolution biologique et transmission culturelle, si bien que l'alternative individu / société n'est d'aucune valeur et qu'isoler un élément - le «cerveau »-pour en faire le point de départ des processus sociaux n'a guère de sens. Parler de «cerveau social», comme le fait Cordonier, est donc équivoque. Il aurait été plus heuristique de se référer à l'idée développée par Chapais selon laquelle il existe une sorte de structure sociale «profonde » humaine dont le cœur réside dans les familles monogames, d'où résultent la parenté patrilinéaire et des systèmes d'alliance, et dont les caractéristiques - reflétant la biologie de notre espèce - apparaissent par comparaison avec la structure sociale de type chimpanzé ou d'autres sociétés primates et vont de pair avec certaines facultés cognitives.
} 
taine de pages, et arriver jusqu'au milieu du troisième chapitre, pour commencer à comprendre de quoi il en retourne.

Le troisième chapitre, intitulé «La cognition sociale humaine », est de loin le plus long du volume. Il faut dissiper d'emblée une équivoque possible : l'adjectif «sociale», ici, renvoie non pas à des processus de transmission intergénérationnelle des instruments humains d'orientation et de communication, à des processus d'apprentissage, mais aux interactions quotidiennes, aux relations interindividuelles hic et nunc. Selon Kaufmann et Clément, les interactions humaines se coulent dans le moule de formes élémentaires (relations de dominance, d'échange, de soin et d'affiliation, auxquelles Cordonier ajoute les relations d'alliance, de coopération et d'antagonisme). Or, pour un sociologue, la « rationalité » du comportement et de l'économie psychique des agents n'est compréhensible qu'à la condition de reconstituer les structures du réseau d'interdépendances et du système de domination qui l'imposent et la rendent, pour ainsi dire, «naturelle ». Prenons l'exemple du courtisan étudié par Norbert Elias dans La Société de $\operatorname{cour}^{18}$ : on ne voit pas quel «type de relation» préétabli aurait aidé, mieux que le patient travail de reconstruction empirique des liens d'interdépendances constitutifs de la "société de cour », à appréhender le souci obsessionnel du statut et du prestige, du paraître et des formes, qui gouverne son attitude et sa structure de personnalité. De même qu'on ne saurait guère discerner, à s'en tenir à ces formes élémentaires de relations sociales, par quels mécanismes la domination sociale des hommes sur les femmes peut en venir à s'inverser, par exemple en période de crise thérapeutique dans le contexte sorcellaire du Bocage ${ }^{19}$.

Non seulement, pour Kaufmann et Clément, les interactions humaines sont susceptibles d'être ramenées à un certain type de relations sociales (dominance, échange, soin ou affiliation), mais, en plus, il se pourrait bien que de nombreuses «institutions culturelles» résultent du «travail proprement humain de symbolisation des formes sociales élémentaires. Par exemple, l'institution du "don contredon" consisterait en une élaboration symbolique particulière de la relation d'échange, la Nation tirerait son origine de la relation d'appartenance, la famille prendrait racine sur la relation de soin, la division sociale du travail serait construite à partir de la relation de coopération, etc. » (p. 153) Le propos de Cordonier, qui consistait largement en un résumé précis et pédagogique de travaux sérieux (Frans de Waal, Philippe Rochat ou Lawrence Hirschfeld), bascule ici dans l'à peu-près et l'indémontrable. On n'est pas très éloigné du paradoxe de l'œuf et de la poule : qui, des relations sociales élémentaires ou des formes symboliques, est arrivé en premier? Le problème d'une typologie aussi générale est qu'on peut y faire entrer n'importe quel fait empirique, sans aucune possibilité de vérification et de contrôle des rapprochements intuitifs ainsi opérés. Cela ne nous ramène pas seulement aux apories de la sociologie formelle d'un Simmel, contre laquelle le jeune Elias jeta les bases d'une théorie sociologique des interdépendances propre à favoriser la recherche empirique et la cumulativité scientifique ${ }^{20}$. Cela nous place, de surcroît, au plus loin d'une vision dynamique et structurée des processus, et, notamment, du travail d'enquête et de reconstruction logique des ordres séquentiels en vertu duquel il est permis d'affirmer que "l'évolution des liens sexuels stables a obligatoirement précédé l'évolution des premières alliances intergroupes ${ }^{21}$, ou, à l'instar du psychologue John Flavell (non cité par Cordonier), que les enfants de trois ans n'ont pas les même capacités de compréhension de la distinction apparence / réalité que les enfants de cinq ans ${ }^{22}$.

Aucune typologie ne saurait sans doute se substituer au travail d'enquête empirique, de problématisation et de conceptualisation constitutif de l'approche développementale des processus sociaux, des processus d'incorporation des structures sociales, des modes de construction psycho-socio-génétique du rapport au monde ou du style relationnel de chacun. Cette approche paraît étrangère à l'horizon de pensée des tenants du «naturalisme social ». 11 faudrait une enquête approfondie pour saisir adéquatement les propriétés de leur trajectoire ainsi que les dispositions et ressources qui les ont amenés à se positionner dans un domaine de recherche, celui de la «théorie de l'esprit» et de la «cognition so-

\footnotetext{
${ }^{18}$ Norbert Elias, La société de cour, trad. de l'allemand par Pierre Kamnitzer et Jeanne Etoré, préface de Roger Chartier, Paris, Flammarion, 1985 [1974].

19 Jeanne Favret-Saada, Désorceler, Paris, L’Olivier, 2009. Voir notamment la section intitulée «La thérapie comme travail domestique ».

${ }^{20}$ Voir Marc Joly, La révolution sociologique. De la naissance d'un régime de pensée scientifique à la crise de la philosophie (19 $-20^{e}$ siècle), Paris, La Découverte, 2017.p. 271-276.

${ }^{21}$ Bernard Chapais, Aux origines de la société humaine, op. cit., p. 46.

${ }^{22}$ John H. Flavell, « The development of children's knowledge about the appearance-reality distinction », American Psychologist, vol. 41, n 4, 1986, p. 418-425.
} 
ciale », initialement travaillé par des psychologues du développement cognitif ${ }^{23}$; ou dans quels espaces de controverses et sous l'influence de quels espaces de positions et de prises de position ils ont conçu leurs propositions «naturalistes $»^{24}$.

Le lecteur de La nature du social, ne disposant pas de ces informations, en est réduit à essayer de deviner en quoi consiste précisément cette «cognition sociale » que les humains partageraient avec les autres primates (tout en se différenciant de ces derniers par leur capacité à conférer une «dimension culturelle » aux « relations sociales »), qui ne se réduirait pas à la «théorie de l'esprit » et qui se manifesterait, on ne sait trop comment, «chez les enfants de moins d'un an déjà » (p. 159), pour rester par la suite "globalement inchangée » (p. 218). Si Laurent Cordonier évoque sa participation à des expériences de psychologie de l'enfance dans un laboratoire aux États-Unis, on ignore, à s'en tenir au texte, quelles démarches de recherche et quels protocoles d'enquête fondent de telles propositions. Ce qui n'est pas clair, c'est la question de savoir comment, de l'idée recevable selon laquelle les êtres humains apprennent, sur la base de mécanismes universels de computation mentale, des normes de comportement propres à une société ou à un groupe social donnés, on passe à la thèse d'une possession innée de représentations des relations sociales (c'est la « sociologie nä̈ve », que les défenseurs $\mathrm{du}$ «naturalisme social» empruntent à Hirschfeld). Ce qui est encore moins clair, c'est la question de savoir comment les institutions dites «culturelles» procèdent des relations dites «sociales ». Mettre en lien la famille avec la «relation de soin» ou la nation avec la «relation d'appartenance », force est une nouvelle fois de le souligner, n'apporte pas grand-chose à la connaissance des processus sociaux ; la proposition est si générale qu'elle en devient invérifiable ${ }^{25}$.

Disant cela, nous ne sommes pas moins convaincus que Laurent Cordonier de la nécessité de situer la sociologie au sein d'un régime conceptuel et d'un ordre de connaissance plus vastes que ses frontières disciplinaires établies (qui, en tout état de cause, sont historiquement fluctuantes). Et nous partagerions volontiers le rêve d'une " communauté sociologique vivante et en interaction constante avec les autres sciences susceptibles d'éclairer le comportement humain $»^{26}$. Sauf qu'il convient de procéder de manière ordonnée, et de ne pas perdre en cours de route deux impératifs fondamentaux :

1. ne jamais cesser de produire des connaissances sur notre réalité de référence, en s'en tenant, en pratique, aux quelques principes qui donnent sens au projet de science sociale unifiée (pluralité des méthodes, posture de recherche processuelle, relationnelle et comparatiste, réflexivité) ;

2. poser et étudier systématiquement en termes sociologiques le problème des relations interdisciplinaires et des hiérarchies académiques.

S'il fallait s'essayer à une définition générale, on pourrait dire que la sociologie est d'abord la science des liens sociogénétiques, c'est-à-dire des transformations continues à travers lesquelles des configurations dotées de certaines caractéristiques donnent naissance à des configurations structurées différem-

\footnotetext{
${ }^{23}$ Un représentant de ce domaine aussi important que John Flavell, rappelons-le, est absent du livre de Cordonier : ce n'est peut-être pas sans rapport avec la posture piagétienne du psychologue états-unien.

${ }^{24} \mathrm{Qu}$ 'on prenne en compte les disciplines de formation, les références mobilisées, les appartenances institutionnelles, les liens de dépendance ou les différences de génération, tout donne le sentiment, à la lecture du dossier de la revue en ligne SociologieS sur «Le naturalisme social» (2011-2012) (https://journals.openedition.org/sociologies/3594), d'avoir affaire à une dispute de famille. Louis Quéré, qui signe l'un des deux articles critiques, était par exemple le co-directeur de thèse de Laurence Kaufmann et avait auparavant dirigé à l'EHESS son mémoire de DEA en sociologie. Fabrice Clément a également soutenu sa thèse à l'EHESS (Institut Jean Nicod), sous la direction de Dan Sperber. Vincent Descombes était membre des deux jurys de thèse. L'horizon de référence est donc celui d'un dialogue entre la philosophie (à dominante analytique) et les sciences sociales (orientées vers le pragmatisme).

${ }^{25}$ Il faudrait peut-être déjà, avant de postuler quelque capacité innée d'interaction sociale ou d'orientation au sein de formes typiques de relations sociales dûment repérées, approfondir par exemple la notion d'attachement de John Bowlby, qui repose sur des recherches précises, au croisement de l'éthologie et de la psychanalyse. Voir par exemple John Bowlby, «La psychanalyse : une science naturelle » [1980], dans Le lien, la psychanalyse et l'art d'être parent, trad. de l'anglais par Yvane Wiart, Paris, Albin Michel, 2011, p. 93-118.

${ }^{26}$ Fabrice Clément, « Sciences de l'esprit et sciences sociales : des liaisons dangereuses ? , SociologieS [En ligne], Débats, Le naturalisme social, mis en ligne le 09 mai 2012, http://journals.openedition.org/sociologies/3980.
} 
ment $^{27}$. C'est en tant que telle qu'elle a été amenée à parachever, autour de 1900, la formation d'un régime de pensée historicisant et objectivant de l'humanité - un régime bio-psycho-sociologique cadrant la recherche et les représentations. Sa réalité de référence, ce sont les sociétés humaines en développement et leurs membres interdépendants par nature et selon des modes spécifiques indissociables de dispositifs de contrôle de la nature, des rapports sociaux et des comportements individuels (l'économie psychique individuelle se structurant en rapport avec ces dispositifs de contrôle ou de contrainte). Dans cette perspective, la distinction du « culturel » et du « social », à laquelle Cordonier revient sans cesse, est tout à fait secondaire.

Il n'est pas déraisonnable de penser que les sociologues ont besoin en premier lieu de pouvoir travailler dans le cadre d'une théorie des processus sociaux conçue en termes de changements configurationnels et de genèse des structures (et non pas en termes strictement «émergentistes », qui recèlent tant de fausses pistes, comme le réductionnisme ou le causalisme, ou de fausses-oppositions: dualisme / monisme, individualisme/holisme, etc.). Laurent Cordonier et les tenants du «naturalisme social » touchent certes juste sur un point : les sociologues ont aussi besoin d'autres repères. Ils doivent pouvoir se référer à un modèle de développement bio-psycho-sociologique du sujet générique; ils doivent pouvoir se référer à un modèle dynamique du développement des «sciences humaines et sociales » comme sciences reliées les unes aux autres au sein d'un même régime. On peut néanmoins douter que le «naturalisme social» fournisse de tels repères ${ }^{28}$. Et il reste que le plus important, en sociologie comme en toute science, est d'être capable de formuler des problèmes circonscrits de manière pertinente et de les résoudre conformément à des principes théorico-empiriques éprouvés.

\section{Que peut le naturalisme social pour la sociologie ?}

\section{Une présentation caricaturale de la discipline sociologique}

«Tout paradigme sociologique prend nécessairement appui sur une certaine élaboration théorique de la nature humaine et du fonctionnement de l'esprit humain » (p. 241), écrit Laurent Cordonier en ouverture du chapitre 5 consacré aux liens entre « sciences cognitives et théories sociologiques ». Présentant successivement ce qu'il considère être les « deux grands courants historiques de la tradition sociologique » (p. 241-242), à savoir l'individualisme méthodologique d'une part et le « holisme méthodologique ( (ou «holisme-dispositionnalisme») d'autre part, il indique ensuite que chacune de ces branches peut «s'articuler avec une approche naturaliste » (p. 242).

Il n'échappera cependant à personne que «prendre appui sur» et «s'articuler avec » désignent des relations de nature différente. Se servir d'une conception extérieure de «l'esprit» pour (pouvoir?) faire de la sociologie, ce n'est pas la même chose que d'œuvrer à l'intérieur d'un seul et même régime de pensée à la connaissance scientifique de la nature humaine. Durkheim, de ses premières prises de position à son dernier grand ouvrage, Les formes élémentaires de la vie religieuse, n'a jamais cessé de s'inscrire dans la perspective d' «une nouvelle manière d'expliquer l'homme ${ }^{29}$. Il a toujours cherché à combiner de la manière la plus rigoureuse possible, dans l'espace du pensable de son temps, les principes suivants : 1 . distanciation et visée d'une connaissance congruente à la réalité $; 2$. conceptualisation des faits sociaux; 3 . élaboration d'une nouvelle image de l'humanité ${ }^{30}$. Cela « ouvre » la sociologie, mais pas n'importe comment ni à tous les vents. Sous cet angle, seule la maîtrise des méthodes adaptées à l'étude et à la conceptualisation des structures et processus constitutifs de la réalité de référence de la sociologie est susceptible de favoriser la compréhension des implications épistémologiques et anthropologiques de cette nouvelle science inséparable de la biologie et de la psychologie.

\footnotetext{
${ }^{27} \mathrm{C}$ 'est très exactement une démarche de ce genre que met en œuvre dans son dernier livre l'économiste Thomas Piketty, en analysant notamment le passage des «sociétés ternaires » aux «sociétés de propriétaires ». Voir Thomas Piketty, Capital et idéologie, Paris, Seuil, 2019.

${ }^{28}$ L'ouvrage La sociologie cognitive dirigé par Fabrice Clément et Laurence Kaufmann, qui comporte certes des éléments stimulants et couvre un large spectre, trahit ainsi une connaissance approximative de l'histoire des sciences humaines et sociales en général, et de l'histoire de la sociologie en particulier. Cela nuit, d'emblée, aux prises de position épistémologiques et aux prétentions théoriques du «naturalisme social ». Voir, en particulier, Fabrice Clément, «L'esprit de la sociologie », in Fabrice Clément et Laurence Kaufmann (dir.), La sociologie cognitive, Paris, Orphys / MSH, 2011, p. 101-133.

${ }^{29}$ Émile Durkheim, Les formes élémentaires de la vie religieuse, Paris, Le Livre de Poche, 1991 [1912], p. 739.

${ }^{30}$ Cette lecture de la sociologie durkheimienne est exposée dans Marc Joly, Après la philosophie, op. cit., en particulier p. 131-192 dans le cadre d'une longue discussion de l'ouvrage de Thomas Hirsch, Le temps des sociétés. D'Émile Durkheim à Marc Bloch (Paris, EHESS, 2016).
} 
Le «néo-durkheimisme » d'un Elias et d'un Bourdieu procède à bien des égards du croisement fécond entre, d'un côté, ces principes fondamentaux et, de l'autre, la psychanalyse et le constructivisme piagétien. Au-delà, les recherches de sociologie culturaliste cognitive, qui se sont notamment développées à l'Université de Rutgers dans le sillage d'Eviatar Zerubavel et de Karen Cerulo, auraient mérité un meilleur sort que l'ignorance totale dans laquelle les tient Cordonier. Aucun de ces divers programmes scientifiques ne saurait se réduire à une «psychologie de sociologues» (p. 242) ou à de simples «postulats psychologiques » (p. 265), ce qui ne manque décidément pas de faire peser de sérieux doutes sur l'idée selon laquelle la «tradition sociologique » se partagerait entre deux «grands courants historiques $\gg . .$.

De même, il est malencontreux de faire ressurgir dans le sillage de Nathalie Heinich les épouvantails des « sociologues critiques» ou des « sociologues du genre » qui, parmi d'autres, auraient « développé une forte méfiance à l'égard de tout ce qui pourrait tenir de la "nature" dans la vie sociale de l'être humain» (p. 256). Cordonier ne donne aucun exemple témoignant de cette supposée "forte méfiance ». Outre, répétons-le, que le statut de la «nature » comme catégorie scientifique requiert d'être historicisé et éventuellement discuté, un simple survol de la littérature sur le genre - par ailleurs hétérogène, comme toute littérature scientifique - suffit à constater que les processus biologiques sont au cour des discussions ${ }^{31}$ (et pas uniquement pour faire l'objet d'une «disqualification », comme en témoigne par exemple l'approche défendue par Françoise Héritier).

Enfin, on ne voit pas en quoi consiste «l'ontologie physicaliste des sciences contemporaines » qui empêcherait de souscrire à un supposé «holisme ontologique » (p. 255). Des modèles théoriques multidimensionnels s'imposant progressivement dans toutes les sciences, physique comprise ${ }^{32}$, il n'est pas du tout certain que le «physicalisme » (p. 261) règne sans partage dans le champ scientifique. Pour ne donner qu'un seul exemple, Jerry A. Fodor, dont la thèse sur la «modularité de l'esprit » est aujourd'hui parmi les plus influentes dans les sciences cognitives, s'est précisément opposé à l'imposition générale du physicalisme ${ }^{33}$.

Quoi qu'il en soit, les schèmes comportementaux des êtres humains n'étant ni des schèmes spécifiques innés (c'est-à-dire des «instincts ») ni dissociables de la position sociale qu'ils occupent, il convient, semble-t-il, de toujours reconstruire des espaces de positions avant de pouvoir dire quoi que ce soit de pertinent sur les comportements individuels. Ce principe de précaution méthodologique n'est cependant pas synonyme d'adhésion à une quelconque «thèse de l'existence d'une réelle autonomie ontologique du social » (p. 261). De fait, Laurent Cordonier donne la très nette impression de ne pas pouvoir s'affranchir de la vision suivant laquelle il y aurait, d'abord, les individus, avec leur héritage biologique et leurs invariants cognitifs, et, ensuite, les phénomènes sociaux, résultant de leurs actions. À cette vision statique et dichotomique s'oppose la vision processuelle-relationnelle, celle de l'individuprocessus comme partie prenante dès sa naissance de réseaux d'interdépendances constitutifs de processus de développement social possédant leur ordre propre et leurs structures intrinsèques.

\section{Nier coûte que coûte l'importance de la socialisation familiale}

Selon l'auteur, "s'intéresser empiriquement au fonctionnement de l'esprit humain, c'est nécessairement enquêter sur la question de la nature humaine, cette nature héritée de notre évolution biologique et dont nous partageons de très nombreux éléments avec nos cousins non humains. » (p. 256) Notre lignée s'étant séparée il y a six à sept millions d'années des chimpanzés, nul doute qu'il aurait été plus prudent d'évoquer « certains traits » plutôt que de «très nombreux éléments ». La question est en tout cas de savoir dans quelle mesure et selon quelles finalités théoriques explicatives il est utile de parler d'《 instincts sociaux ». À en croire l'auteur, deux d'entre eux, le besoin d'appartenance à un groupe et la préférence pour la similarité, auraient directement favorisé le développement de mécanismes affilia-

\footnotetext{
${ }^{31}$ Voir Juliette Rennes (dir.), Encyclopédie critique du genre, Paris, La Découverte, 2016.

${ }^{32}$ Voir Nancy Cartwright, The Dappled World: A Study of the Boundaries of Science, Cambridge, Cambridge University Press, 1999 ; Norbert Elias, Théorie des symboles, trad. de l'anglais par Damien et Marie-Blanche Audollent, révisé, édité et présenté par Marc Joly, Paris, Seuil, 2015, p. 243-244.

${ }^{33}$ L'ensemble de son échafaudage théorique repose en effet sur le postulat de la multiréalisabilité : qu'un même état mental, comme la peur, puisse s'exprimer chez des espèces dont la structure cérébrale est pourtant différente, montrerait que les processus cognitifs ne sont pas réductibles à des substrats physiques. Voir par exemple Jerry A. Fodor, « Special sciences (or: the disunity of science as a working hypothesis) », Synthese, vol. 28, ${ }^{\circ} 2$, 1974, p. 97-115.
} 
tifs naturels qui éclaireraient, à leur tour, les rouages de la socialisation : il s'agirait de l'imitation et du conformisme (qui sont exposés avec une précision remarquable, p. 170-188).

Dans quelle mesure cette idée permet-elle de donner quelque contenu à la notion de « cognition sociale »? L'analyse du traitement par Cordonier du concept d'habitus peut nous aider à trancher la question. Et disons-le d'emblée : l'auteur ne semble pas voir que la souplesse des points de jonction constructivistes et psychanalytiques de cette notion sociologique laisse grande ouverte la possibilité de croisements plus affinés avec, notamment, les neurosciences ${ }^{34}$. Son point de vue aurait également été plus équilibré s'il avait mieux explicité ses références psychologiques et s'il avait évité de reprendre à son compte des prises de position représentatives de certaines évolutions non analysées des champs concernés (en particulier aux États-Unis).

C'est flagrant dans l'importance qu'il accorde aux écrits de la psychologue Judith Rich Harris (19382018), dont la thèse controversée, concluant à l'absence totale d'influence de l'éducation parentale sur le développement de la personnalité, est fréquemment mobilisée par les tenants d'une vision biologisante des comportements humains ${ }^{35}$.

Dans son ouvrage intitule The Nurture Assumption: Why Children Turn out the Way they Do (Pourquoi nos enfants deviennent ce qu'ils sont, en traduction française), cette chercheuse indépendante, ancienne rédactrice de manuels universitaires, met en scène son abandon de la doxa psychanalytique et psychologique développementale, et sa pénitence au Canossa de la génétique du comportement. Elle se fait fort de déployer une argumentation dont la structure conduit à ce que l'éducation parentale sorte fatalement perdante. D'un côté, son poids est nul face aux effets de l'hérédité (laquelle renverse l'influence parent $\rightarrow$ enfant en une influence enfant $\rightarrow$ parent, la personnalité innée du premier «causant » le comportement du second et contribuant ainsi au renforcement des dispositions originelles et à la différenciation des fratries). De l'autre, elle ne pèse rien par comparaison à l'influence du seul environnement qu'il est possible d'abstraire sans coup férir de la génétique et, donc, de reconnaitre en tant que tel dans la balance «nature »/ «culture », «inné » / «acquis »: l'environnement du groupe des pairs. Ainsi pris en étau entre l'hérédité et l'environnement des pairs, l'environnement familial est réduit à néant.

Cette structure argumentative est manifestement spécieuse. Selon Harris, les similitudes entre la personnalité, les conduites et les valeurs des parents et celles de leurs enfants s'expliqueraient par tout sauf par l'éducation parentale, et il en irait de même de leurs dissemblances. C'est évidemment hautement improbable. Il est inutile d'entrer dans le détail de cette argumentation, qui allègue de nombreuses preuves difficilement vérifiables, puisqu'elles mesurent pour la plupart et de manière assez floue des «traits de personnalité ». En matière de «personnalité », les parents n'exerceraient aucun effet prévisible sur leurs enfants. Or il est impossible d'en dire autant du point de vue de leur réussite scolaire et de la reproduction des positions sociales. Conceptuellement, Harris échoue à rendre justice à la complexité de l'enchevêtrement qui singularise tout parcours individuel pris isolément, et qui, en même temps, épouse de bout en bout la réalité structurée d'une société en développement et met en jeu les contraintes inhérentes au développement ontogénétique. Obsédée à l'idée de démêler en termes définitifs les causes et les effets, la psychologue écrase totalement cette complexité. À notre naissance, on prend place dans des configurations qui sont en mouvement constant - mais pas n'importe comment. Notre développement voit s'entremêler maturation biologique et expériences subjectives dans des réseaux de relations qui transcendent les existences individuelles. Apprenant le langage d'une

\footnotetext{
${ }^{34}$ Voir, entre autres : Karen A. Cerulo, « Mining the intersections of cognitive sociology and neuroscience », Poetics, vol. 38, n 2, 2010, p. 115-132 ; Marc Joly, Pour Bourdieu, Paris, CNRS Éditions, 2018, p. 167-182 ; Omar Lizardo, «The cognitive origins of Bourdieu's habitus », Journal for the Theory of Social Behaviour, vol. 34, n 4, 2004, p. 375-401; Arnaud Saint-Martin, «Le danger sociologique? Un feu de paille », Zilsel, ${ }^{\circ} 3$, 2018, p. 427 ; Loïc Wacquant, «Putting Habitus in its Place: Rejoinder to the Symposium », Body \& Society, vol. $20, \mathrm{n}^{\circ} 2,2014$, p. 129.

${ }^{35} \mathrm{C}$ 'est le cas, par exemple, des criminologues biosociaux qui, sur le fondement notamment des résultats empiriques issus des twin studies, font valoir une conception pro-génétique des comportements déviants aux termes de laquelle la biologie serait causalement prépondérante. Sans surprise, une recherche bibliométrique effectuée sur le Web of Science révèle effectivement que l'ouvrage de Judith Harris, The Nurture Assumption, est fréquemment cité par ces derniers : depuis 2000, 65 articles publiés dans des revues de criminologie ont cité cet ouvrage. Une partie importante de ces articles émane des principaux représentants de la criminologie biosociale pro-génétique : mentionnons notamment Kevin Beaver, qui se trouve être "Judith Rich Harris Professor of Criminology » à Florida State University (signataire ou cosignataire de 31 articles) ; J. C. Barnes (16 articles) ; John Paul Wright (11 articles); Brian Boutwell (10 articles); ou encore Matt DeLisi (5 articles).
} 
société spécifique ou intégrant un modèle socialement sanctionné d'autorégulation pulsionnelle, chacun traverse, à son rythme, des changements de palier incontournables, aux conséquences difficilement réversibles ${ }^{36}$. Dans la majorité des cas, les parents introduisent à cet enchevêtrement ; et cet enchevêtrement lui-même ne laisse pas de reconduire aux parents. Nier l'«éducation parentale » n'a donc aucun sens, et il n'est pas étonnant que la psychologue finisse par livrer des réflexions sur les styles d'éducation d'une grande pauvreté, méconnaissant les rapports sociaux différenciés au langage ${ }^{37}$.

Laurent Cordonier n'en prolonge pas moins (p. 291-297) l'approche de Judith Harris afin de critiquer l'idée d'un «déterminisme familial qui condamnerait, statistiquement parlant, les enfants ou les adolescents issus des milieux les plus modestes à moins bien réussir leur parcours scolaire que les autres» (p. 316). Tout se passe comme s'il s'était demandé comment procéder pour contrecarrer la démonstration bourdieusienne selon laquelle, les familles les mieux dotées sur le plan du capital culturel transmettant des savoirs et des savoir-faire bénéfiques dans le système scolaire, celui-ci contribue à la reproduction des classes selon un mécanisme statistique. Du point de vue des enfants, cela se traduit par des probabilités différentes de réussite. Inspiré par Harris, Cordonier s'attache par contraste à inventer un «habitus scolaire » et à déconnecter celui-ci des savoirs et savoir-faire transmis par la famille et susceptibles de cadrer plus ou moins fortement les expériences scolaires. Cet «habitus scolaire », les enfants l'incorporeraient « en s'alignant par imitation et conformisme sur les dispositions pratiques, les attitudes et les intérêts de leurs amis d'école»(p. 317). On comprend l'intérêt de l'auteur pour la socialisation auprès des pairs : elle lui paraît mettre en jeu les deux mécanismes affiliatifs sous-jacents de l'imitation et du conformisme. Dès lors, il suffirait de faire en sorte que les enfants issus des milieux populaires puissent intégrer des groupes de pairs majoritairement composés d'élèves «mieux préparés et adaptés [...] aux exigences scolaires » (p. 317) pour qu'ils acquièrent les dispositions valorisées par le système éducatif... dispositions qui trouvent leur origine dans certaines familles. Ces contorsions n'étaient pas de trop pour congédier un bien obscur «déterminisme familial $»^{38} \ldots$

Il n'est d'ailleurs que de se pencher sur les stratégies déployées par les parents des classes moyennes et supérieures afin de contourner la carte scolaire pour percevoir la naïveté de l'argument ${ }^{39}$. Car ces stratégies - un véritable sport national, en France - se déploient précisément au moment de l'entrée au collège, à l'âge où les enfants sont censés acquérir leur « habitus » en dehors de la sphère parentale : il s'avère que l'influence des parents sur leur destinée est alors plus forte et directe que jamais. Cordonier n'est pas loin de le reconnaître - tout en se contentant d'évoquer la ségrégation sociale des zones d'habitation - lorsqu'il défend justement l'idée d' « habitus de classe ${ }^{40}$.

\footnotetext{
${ }^{36}$ Voir Howard S. Becker, The Tricks of the Trade. How to Think about Your Research While You're Doing It, Chicago, Chicago University Press, 1998 (en particulier la section intitulée «What is a nice girl like you doing in a place like this? »).

37 «Si la culture du groupe de pairs diffère de celle des parents, c'est toujours la première qui l'emporte. L'enfant d'immigrés ou de parents sourds apprend immanquablement la langue de ses pairs et la préfère à celle qu'il a pu apprendre chez lui. Elle devient sa langue maternelle » (Judith Rich Harris, Pourquoi nos enfants deviennent ce qu'ils sont. De la véritable influence des parents sur la personnalité de leurs enfants, trad. de l'anglais (États-Unis) par Odile Demange, Claude Farny et Bella Arman, Paris, Pocket, 2004 [1999], p. 559).

${ }^{38}$ La question de savoir comment un enfant peu préparé dans sa famille à répondre aux attentes du système scolaire s'intègre effectivement dans un groupe de pairs composé majoritairement d'élèves dont les savoirs et savoirfaire sont ajustés par anticipation à l'école a été différemment traitée en sociologie et peut donc bénéficier d'une multiplicité d'éclairages. On pense par exemple à l'ouvrage de Paul Pasquali, Passer les frontières sociales. Comment les «filières d'élite » entrouvrent leurs portes, Paris, Fayard, 2014. Plus récemment, les politistes Laurent Bonelli et Fabien Carrié ont apporté à propos de l'engagement de certains jeunes dans une radicalité islamique «utopique » des éléments qui devraient donner à réfléchir. Selon eux, le fait, pour un jeune issu d'un milieu modeste et d'une famille immigrée, de se trouver projeté dans un lycée d'élite, et soumis à une intense compétition scolaire après un fort investissement au collège, peut être très difficile à vivre et engendrer des frustrations mortifères. Voir Laurent Bonelli, Fabien Carrié, La Fabrique de la radicalité. Une sociologie des jeunes djihadistes français, Paris, Seuil, 2018. Voir également Bernard Lahire (dir.), Enfances de classe. De l'inégalité parmi les enfants, Paris, Seuil, 2019.

${ }^{39}$ Pierre Merle, «La carte scolaire et son assouplissement. Politique de mixité sociale ou de ghettoïsation des établissements ?», Sociologie, vol. 2, n 1, 2011, p. 37-50.

${ }^{40}$ Il sait aussi parfois être relativement prudent dans ses formulations : « La disposition à l'engagement scolaire d'un élève est bel et bien - en partie au moins - le produit du processus de socialisation par son groupe de pairs »
} 
Raisonner processuellement permet d'éviter l'alternative « ou les parents / ou les pairs ». Les uns et les autres jouent dans la fabrication de l'« habitus ». Du reste, à suivre Cordonier, les agents sociaux se caractériseraient non pas par un habitus, mais par une multiplicité d'habitus entre lesquels ils jongleraient. Par exemple, les enfants disposeraient d'un «habitus scolaire » et de divers «habitus extrascolaires » (p. 304), mais aussi d'un ou de plusieurs habitus « publics» (p. 305), ce qui devrait signifier qu'il en existe aussi de " privés ${ }^{41}$. De l'habitus, on perd ainsi de vue le caractère organisateur des pratiques de l'individu - un individu actif et agi selon les « lois » de probabilités attachées aux positions sociales. L'habitus n'est pas un concept du même ordre que le « rôle » ou la «persona » (dont fait usage Harris). C'est un concept dynamique et intégratif, empiriquement fondé, qui aide à penser $e t$ à expliquer le caractère organisateur des pratiques individuelles ainsi que les corrélations statistiques entretenues par ces dernières avec les positions sociales correspondantes ${ }^{42}$.

\section{«Intégration "... ou désintégration?}

On conclura sur l'aspect le plus original du livre de Laurent Cordonier - celui qui illustre le mieux la problématique de l'interdisciplinarité. Dans le chapitre 5, l'auteur détaille en effet une expérience de psychologie sociale menée à l'Institut des sciences cognitives du CNRS à Lyon (Institut Marc Jeannerod), à laquelle il a contribué.

Cette expérience, qui a fait l'objet d'une publication dans la revue interdisciplinaire Interaction Stu$\operatorname{dies}^{43}$, reposait sur le protocole suivant. Dans le but de tester l'effet de l'impact des hiérarchies sociales sur les réactions psychophysiologiques, trente-six individus, de sexe masculin, droitiers et étudiants en licence, ont été répartis égalitairement en deux groupes; et chaque groupe s'est vu présenter la même personne - un complice des chercheurs -, mais sous deux étiquettes différentes reflétant, respectivement, un «bas statut» et un «haut statut» (p. 271). Afin de «tromper » les participants, conscients de prendre part à une expérimentation, les chercheurs expliquèrent à chacun "qu'il s'apprêtait à prendre part à une étude sur les réponses somatiques aux interactions sociales », avant de l'équiper de «capteurs mesurant son activité physiologique » (p. 271). Le test de l'effet du prestige social intervenait cependant réellement lorsque l'expérimentateur faisait savoir qu'une personne allait venir vérifier le bon fonctionnement des capteurs et qu'il importait de ne pas bouger pendant cette opération : pour la première moitié des individus, le complice était «Erwan, un aide-soignant»; pour la deuxième, le même complice, revêtu à peu près de la même blouse blanche et se comportant identiquement, était le «docteur Wibaux, un neurochirurgien» (p. 271). C'est lors de ces interactions que des mesures de la conductance électrodermale et de la fréquence cardiaque des participants furent prélevées.

À partir du résultat de ces mesures, Cordonier conclut que «la réponse psychophysiologique des participants testés dans la condition "bas statut" indique qu'ils se contentèrent de prêter attention à l'approche de l'aide-soignant», tandis que «les participants testés dans la condition "haut statut" affichèrent, pour leur part, une réaction psychophysiologique bien plus forte : ils se préparèrent automatiquement à réagir physiquement à l'approche du neurochirurgien » (p. 274). Et il estime que cela corrobore l'hypothèse de Bourdieu selon laquelle l'habitus s'incorpore et devient comme une

(p. 311). Une observation précise pourrait en effet conduire à rencontrer des enfants dont l'engagement scolaire est contrarié ou au contraire stimulé par des pairs aux propriétés sociales différentes. De tels processus n'en dépendent pas moins de la répartition différenciée du capital culturel et de la corrélation attestée entre l'origine sociale et la réussite scolaire grâce à la transmission familiale.

${ }^{41}$ Cela dit, Laurent Cordonier ne veut pas entendre parler d'《habitus familial ». Il va jusqu'à écrire : «La famille est-elle pour autant le lieu où ces derniers [les enfants] vont forger leur habitus ? Rien n'est moins sûr » (p. 291). En somme, là où Harris parlait de «personnalité » et déniait toute influence familiale, Cordonier parle d'« habitus » tout en opérant le même déni, ce qui apparaît paradoxalement encore plus intenable. En se mettant dans les pas de Harris, il passe par pertes et profits tous les acquis de la psychanalyse et de la psychologie développementale de l'enfance qui pourraient donner les moyens d'élaborer un modèle du développement ontogénétique empiriquement fondé et mobilisable par les sociologues.

${ }^{42}$ Il ne devrait pas être nécessaire de préciser qu'une théorie est par définition explicative. Cordonier distingue « théorie » et «explication» (le chapitre 5 est intitulé «Sciences cognitives et théories sociologiques », le chapitre 6, «Sciences cognitives et explications sociologiques»). Or les théories sociologiques ne visent à rien d'autre qu'à expliquer des phénomènes sociaux.

${ }^{43}$ Laurent Cordonier, Audrey Breton, Emmanuel Trouche et Jean-Baptiste Van der Henst, « Does prestige affect us physiologically? A study in an interactional context », Interaction Studies, vol. 18, n 2, 2017, p. 214-233. 
« seconde nature ». S'il n'était pas «possible d'observer des traces (psycho)physiologiques de cette incorporation » (p. 269), alors il ne s'agirait que d'une «simple métaphore» (p. 269)...

Rappelons, tout d'abord, que le concept d'habitus procède d'une construction scientifique qui ne le réduit pas fatalement au statut de « simple métaphore » en l'absence de traces physiologiques attestées de manière décisive. Et essayons, ensuite, de démêler l'écheveau de la démonstration :

1. on observe des réactions différentes du système nerveux autonome des participants des deux groupes face à un agent qu'ils classent différemment dans l'ordre social - tantôt vers le bas, tantôt vers le haut -, du moins le suppose-t-on ;

2. ces réactions interviennent à un moment précis de l'interaction, durant la «phase d'approche » (p. 273), lorsque le complice des expérimentateurs «s'approchait du participant et s'asseyait à côté de lui » (p. 272) ;

3. cela indiquerait que les individus confrontés au neurochirurgien se sont sentis spontanément placés dans une relation de dominance, ce qui irait dans le sens non seulement de l'existence d'une « sociologie naïve des relations » postulée par le naturalisme social (p. 277), mais de l'hypothèse d'une incorporation de l'habitus (en somme, ils auraient si bien appris à respecter les hiérarchies sociales qu'ils auraient inconsciemment activé des dispositions pratiques incorporées, se préparant soit «à agir d'une manière déférente », soit "à résister à son autorité » [p. 274], comme en témoigne l'augmentation de leur fréquence cardiaque, aux prémices de leur interaction avec un individu censément de statut élevé).

Nous aurions aimé être enthousiastes et pouvoir saluer une belle rencontre de la sociologie avec les neurosciences. Mais, à y regarder de près, nous sommes sceptiques. Une nouvelle fois, c'est la posture de sociologue de Laurent Cordonier qui interroge.

Si on se reporte à l'article original publié avec les membres de l'Institut Marc Jeannerod, on constate que le concept d'habitus n'apparaît pas - et pas davantage le nom de Bourdieu. Cordonier prend soin de préciser, dans une note de bas de page de son livre, que l'interprétation qu'il propose " $n$ 'engage pas les autres auteurs » (p. 274). Il reconnaît lui-même que l'expérience n'a pas été pensée comme un test empirique de l'hypothèse d'une incorporation de l'habitus, et, ce faisant, il est doublement ambigu, puisqu'il signale que tel n'était pas le «but initial» de cette expérience (ce qui pose la question des interprétations a posteriori) et ajoute que c'est la «validité des postulats psychologiques sousjacents à la sociologie bourdieusienne » (p. 270) qui serait après coup en jeu (ce qui ne constitue pas une façon satisfaisante, on le sait, de formuler le problème des relations de complémentarité entre la sociologie et les sciences psychologiques et biologiques).

S'il est permis de douter que le protocole suivi par les expérimentateurs permette de saisir le concept d'habitus dans toute sa complexité, c'est d'abord parce que rien ne laisse penser que Laurent Cordonier - après tout premier signataire de l'article paru dans Interaction Studies - ait su profiter de sa collaboration avec des chercheurs en sciences cognitives pour favoriser la réalisation d'une réelle expérience interdisciplinaire, en orientant l'«expérience » en un sens plus sociologique. C'était peutêtre hors de portée. Il reste qu'il n'était même pas nécessaire d'aller jusqu'à imposer la théorie bourdieusienne à des chercheurs extérieurs au champ sociologique : introduire des variables contrôle supplémentaires qui auraient permis de mieux saisir le positionnement social des participants était-il si difficile ? En l'état, on ne dispose malheureusement d'aucune information sur leurs origines sociales. Il n'a pas été demandé aux participants d'indiquer la situation socioprofessionnelle ou le niveau de revenu de leurs parents, lit-on en conclusion de l'article. Il est également suggéré des variantes de l'expérience avec des participantes femmes ou reposant sur la mise en scène d'autres signaux sociaux de prestige ( $«$ academic distinctions, Ivy League education, or honorific titles ${ }^{44}$ ). Le protocole suivi, qui est celui classiquement mobilisé en psychologie expérimentale, vise à neutraliser le bruit statistique émanant des sujets eux-mêmes. Pour ce faire, on constitue un échantillon d'individus considérés comme semblables et on observe leurs comportements dans un environnement considéré comme neutre: le laboratoire. Cette méthodologie, aussi légitime soit-elle dans certaines branches des sciences cognitives, est-elle pertinente dès lors qu'il s'agit d'" évaluer l'effet [sur le système nerveux autonome] du prestige social, opérationnalisé via le statut professionnel ${ }^{45}$ ? Est-elle compatible avec les principes de méthode et le raisonnement qui ont présidé à l'établissement du couple explicatif position-dispositions ? C'est douteux. La «déférence » et la «résistance » à l'égard de l'autorité ne sontelles pas des dispositions antinomiques dont on ne peut comprendre l'origine et les conditions d'activation qu'en les situant en regard de propriétés, de trajectoires, de positions sociales?

\footnotetext{
${ }^{44}$ Ibid., p. 229.

${ }^{45}$ Ibid., p. 214.
} 
Mais on comprend encore moins que Cordonier n'ait visiblement pas cherché à intégrer dans l'expérience certains réflexes de méthode de base. Certes, ce sont des réflexes «critiques »... Il paraitt toutefois assez évident que, si on se borne à ne faire différer deux conditions expérimentales que par le titre professionnel d'un complice, rien ne permet de conclure décisivement à un effet de prestige social. En l'espèce, rien n'empêche d'imaginer la même expérience, avec des procédés et des résultats identiques, mais menée dans l'idée de mettre en valeur l'angoisse intériorisée du «médecin». L'appellation de «neurochirurgien » n'est pas très engageante ; elle situe davantage l'expérience dans un contexte médical - ce qui peut aussi, en soi, susciter du stress ${ }^{46}$. Notre organisme est constamment plongé dans des situations qui sollicitent différemment nos sens, notre attention, nos affects, etc., en fonction de notre âge, de notre éducation, de nos expériences, de notre personnalité, etc. Cela ouvre la voie à un grand nombre d'interprétations possibles. La condition étudiante ne prédispose-t-elle pas à une plus grande sensibilité à l'égard des positions d'autorité et des «épreuves »? Peut-être nos étudiants en licence se sont-ils imaginés l'espace d'un instant en situation d' " examen », requis de faire bonne impression auprès d'un « professeur »? Plus «basiquement» encore, peut-on dissocier le statut professionnel du genre dès lors qu'il s'agit de mesurer l'effet du prestige social ? N'aurait-il pas fallu, impérativement, créer des conditions expérimentales avec une «complice » femme ${ }^{47}$ ? Et en désignant l'aide-soignant par son seul prénom et le docteur uniquement par son nom, n'a-t-on pas conditionné les sujets de l'expérience ? Enfin, n'aurait-il pas été utile de demander dans un second temps aux participants du groupe «haut statut» pourquoi ils ont automatiquement "sur-réagi » (par comparaison avec les membres de l'autre groupe) quand le docteur Wibaux est entré dans la salle auréolé du statut de «neurochirurgien»? Et réciproquement : les membres du groupe «bas statut » n'auraient-ils pas dû être sondés pour savoir pourquoi ils n'ont eu aucune «réaction»? Une phase d'entretiens semidirectifs aurait sans soute représenté une vraie plus-value sociologique.

Ce n'est pas ergoter ou jouer les rabat-joie que de poser de telles questions. Ce n'est pas davantage être "méfiant » à l'égard de la biologie ou de la psychologie. Bien au contraire. C'est un regret que nous exprimons : les grandes capacités de synthèse, les qualités d'écriture et la foi scientifique de l'auteur auraient mérité d'être mieux employées. Dans un contexte qui n'est pas favorable au développement institutionnel des sciences sociales, il faut être lucide sur l'état réel des rapports de force entre disciplines. Et veiller à ne pas désintégrer les fondamentaux de la recherche sociologique avant d'appeler à intégrer les connaissances et méthodes des «sciences cognitives » ou des neurosciences mainstream dans nos pratiques.

$* * *$

Nous confessions, au début de cette note critique, un certain embarras. Qu'il s'agisse de l'objet de son enquête - «sciences cognitives » ou «sciences de la nature humaine »? - , de son positionnement théorique - le «naturalisme social »-, de sa lecture de l'habitus ou de la nature de sa contribution à des expériences de psychologie expérimentale, l'auteur, avons-nous essayé de montrer, se révèle étonnamment fuyant. Sa bonne foi n'est pas du tout en cause : il est probable qu'il a été mal orienté. Cela contraste avec la précision remarquable de ses résumés de nombreux travaux d'éthologues, de psychologues du développement ou de cognitivistes, et avec son souci, louable, de rendre compréhensible son propos. Du reste, et c'est un signe, autant ses références, s'agissant des « sciences naturelles », sont quasi exclusivement anglophones, autant, concernant la sociologie proprement dite, ses développements sont centrés sur des débats très franco-français - ce qui le conduit à prolonger, en particulier, la dichotomie «individualisme méthodologique»/ «holisme méthodologique», ou à reprocher à d'insaisissables « sociologues critiques » d'être idéologiquement opposés à la discussion de la biologie. Un ancrage international plus ouvert aux sciences sociales aurait favorisé, à n'en pas douter, la mise en œuvre d'une démarche sociologique historicisante et réflexive ${ }^{48}$. C'est, selon nous, l'écueil principal de La nature du social.

\footnotetext{
${ }^{46}$ On peut ainsi se demander ce qu'aurait donné une expérience avec seulement «Erwan » et «le docteur Wibaux $»$ (sans plus d'indications).

${ }^{47}$ Cette possibilité n'est pas évoquée dans La nature du social et congédiée implicitement et de manière expéditive dans l'article, au motif qu'il a été rapporté que « femmes et mâles présentent des sensibilités différentes à la hiérarchie » (ibid., p. 219).

${ }^{48}$ Par exemple, l'étiquette de «sociologie critique » ne recouvre pas les mêmes réalités de part et d'autre de l'Atlantique. La revue états-unienne Critical Sociology se revendique ainsi de la tradition marxiste plutôt que de la sociologie bourdieusienne, comme en témoigne d'ailleurs une recherche rapide sur le Web of Science : le mot-
} 
clé «Bourdieu » apparait dans le titre, le résumé ou les mots-clés de 4 articles publiés dans cette revue, tandis que le mot-clé « Marx » est utilisé dans 38 publications. 